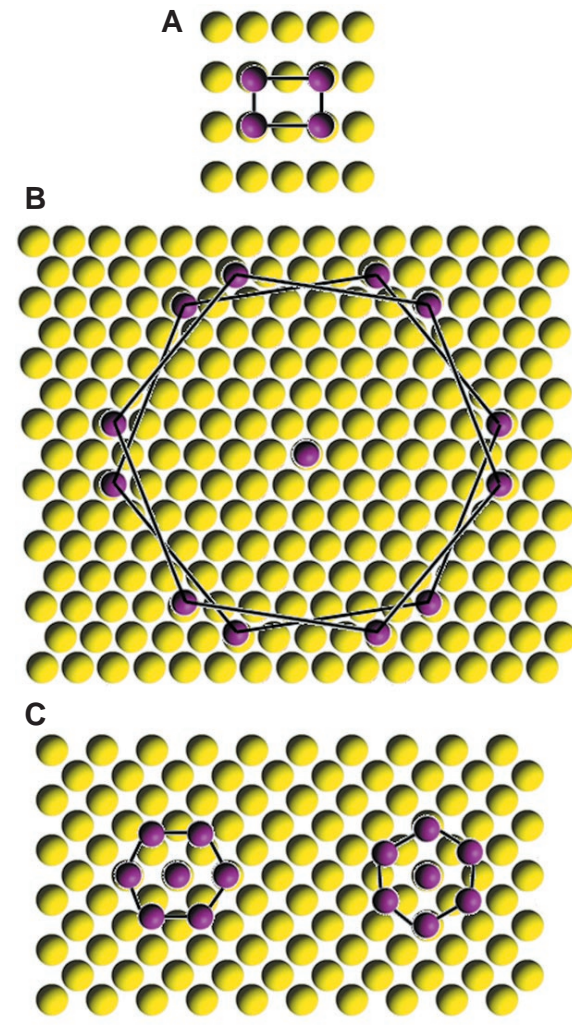

Fig. 4. Interface models for epitaxial growth of $\delta-\mathrm{Bi}_{2} \mathrm{O}_{3}$ on (A) $\mathrm{Au}(110),(\mathrm{B}) \mathrm{Au}(111)$, and (C) $\mathrm{Au}(100)$. The Au substrate atoms are yellow, and the $\mathrm{Bi}$ atoms of $\delta-\mathrm{Bi}_{2} \mathrm{O}_{3}$ are purple. The solid lines indicate unit meshes of the $\delta-\mathrm{Bi}_{2} \mathrm{O}_{3}$ surface nets. The $\delta-\mathrm{Bi}_{2} \mathrm{O}_{3}$ film is rotated $90^{\circ}$ in relation to $\mathrm{Au}(110), \pm 8.9^{\circ}$ in relation to $\mathrm{Au}(111)$, and $0^{\circ}$ and $\pm 30^{\circ}$ in relation to $\mathrm{Au}(100)$. The $(1 \times 1) \mathrm{Bi}_{2} \mathrm{O}_{3}(110)[110] / /$ $(2 \times 1) \mathrm{Au}(110)[100]$ and $\mathrm{Bi}_{2} \mathrm{O}_{3}(111)[121] / /$ $\mathrm{Au}(100)[100]$ coincidence lattices in (A) and (C) both have a mismatch of $-4.2 \%$, and the $(4 \times 4) \mathrm{Bi}_{2} \mathrm{O}_{3}(111) / /\left(\sqrt{31} \times \sqrt{31} R 8.9^{\circ}\right)$ $\mathrm{Au}(111)$ coincidence lattice in (B) has a mismatch of $-2.7 \%$.

(24). Single-crystal films are essential for devices based on superconductor, giant magnetoresistance, thermionic, piezoelectric, and ferroelectric metal oxides because the intrinsic properties of the material, rather than its grain boundaries, can be exploited. The most active crystallographic orientation can also be selected. Our results show that epitaxy can be achieved even for systems with very high lattice mismatch, and they provide a method for producing other nonequilibrium phases that cannot be accessed by traditional thermal processing.

References and Notes

1. J. A. Switzer, M. J. Shane, R. J. Phillips, Science 247 , 444 (1990).

2. J. A. Switzer, R. P. Raffaelle, R. J. Phillips, C.-J. Hung, T. D. Golden, ibid. 258, 1918 (1992).

3. J. A. Switzer et al., ibid. 264, 1573 (1994).

4. R. J. Phillips, T. D. Golden, M. G. Shumsky, E. W. Bohannan, J. A. Switzer, Chem. Mater. 9, 1670 (1997).

5. J. A. Switzer et al., J. Am. Chem. Soc. 120, 3530 (1998).

6. J. A. Switzer, B. M. Maune, E. R. Raub, E. W. Bohannan, J. Phys. Chem. B 103, 395 (1999)
7. P. Shuk, H.-D. Wiemhöfer, U. Guth, W. Göpel, M. Greenblatt, Solid State Ionics 89, 179 (1996).

8, A. M. Azad, S. Larose, S. A. Akbar, J. Mater. Sci. 29, 4135 (1994)

9. For a compilation of work on UPD metals on singlecrystal surfaces, see E. Budevski, G. Staikov, W. J. Lorenz, Electrochemical Phase Formation and Growth: An Introduction to the Initial Stages of Metal Deposition (Wiley-VCH, Weinheim, Germany, 1996), pp. 41-146; ibid., pp. 317-322.

10. , ibid., pp. 201-260.

11. T. P. Moffat, J. Electrochem. Soc. 142, 3767 (1995).

12. D. Lincot et al., Appl. Phys. Lett. 67, 2355 (1995).

13. Y. Golan, J. L. Huchison, I. Rubinstein, G. Hodes, Adv. Mater. 8, 631 (1996).

14. G. S. Hsiao, M. G. Anderson, S. Gorer, D. Harris, R. M. Penner, J. Am. Chem. Soc. 119, 1439 (1997).

15. H. A. Harwig, Z. Anorg. Allg. Chem. 444, 151 (1978).

16. P. D. Battle, C. R. A. Catlow, J. Drennan, A. D. Murray, J. Phys. C. Solid State Phys. 16, L561 (1983).

17. K. Koto, K. Suda, N. Ishizawa, H. Maeda, Solid State lonics 72, 79 (1994).
18. L. E. Depero and L. Sangaletti, J. Solid State Chem. 122, 439 (1996)

19. J. A. Switzer, Ceram. Bull. 66, 1521 (1987).

20. R. A. Van Leeuwen, C.-J. Hung, D. R. Kammler, J. A. Switzer, J. Phys. Chem. 99, 15247 (1995).

21. B. E. Breyfogle, C.-J. Hung, M. G. Shumsky, J. A. Switzer, J. Electrochem. Soc. 143, 2741 (1996).

22. T. D. Golden et al., Chem. Mater. 8, 2499 (1996).

23. J. A. Switzer et al., J. Mater. Res. 13, 909 (1998).

24. We have shown (J. A. Switzer, M. G. Shumsky, E. W. Bohannan, in preparation) in our laboratory that single-crystal films of cuprous oxide $\left(\mathrm{Cu}_{2} \mathrm{O}\right)$ can also be epitaxially electrodeposited onto single-crystal $\mathrm{Au}$. In this case, the lattice mismatch is only $4.4 \%$, and the $\mathrm{Cu}_{2} \mathrm{O}$ overlayer is not rotated in relation to the $\mathrm{Au}$ substrate.

25. This work was supported by the Office of Naval Research grant N00014-96-1-0984, NSF grants CHE9816484 and DMR-9704288, and the University of Missouri Research Board.

12 January 1999; accepted 2 March 1999

\title{
The Fermionic Hanbury Brown and Twiss Experiment
}

\author{
M. Henny, ${ }^{1}$ S. Oberholzer, ${ }^{1}$ C. Strunk, ${ }^{1}$ T. Heinzel, ${ }^{2}$ K. Ensslin, ${ }^{2}$ \\ M. Holland, ${ }^{3}$ C. Schönenberger ${ }^{1 *}$
}

\begin{abstract}
A Hanbury Brown and Twiss experiment for a beam of electrons has been realized in a two-dimensional electron gas in the quantum Hall regime. A metallic split gate serves as a tunable beam splitter to partition the incident beam into transmitted and reflected partial beams. In the nonequilibrium case the fluctuations in the partial beams are shown to be fully anticorrelated, demonstrating that fermions exclude each other. In equilibrium, the crosscorrelation of current fluctuations at two different contacts is also found to be negative and nonzero, provided that a direct transmission exists between the contacts.
\end{abstract}

Experiments aiming at quantum-statistical properties of radiation fields date back to the 1950s when Hanbury Brown and Twiss determined the size of a radio star by measuring the coherence of the observed radiation with an intensity correlation experiment (1). In a follow-up optical tabletop experiment the light of a mercury lamp was partitioned with a beam splitter into a transmitted and reflected beam. The intensity correlation between the two partial beams was found to be positive, demonstrating an enhanced probability for the simultaneous detection of two photons - one in each partial beam $(2,3)$. This is a generic property for a stream of particles obeying Bose-Einstein statistics, in which the particles tend to bunch in clusters. In contrast, antibunching is expected for fermions, because two indistinguishable fermions must exclude each other by the Pauli principle

${ }^{1}$ Institute of Physics, University of Basel, Klingelbergstrasse 82, CH-4056 Basel, Switzerland. ${ }^{2}$ Solid State Physics Laboratory, ETH Zürich, CH-8093, Switzerland. ${ }^{3}$ Department of Electronics, University of Glasgow, Glasgow G12 8QQ, UK.

*To whom correspondence should be addressed: Email: schonenberg@ubaclu.unibas.ch
(Fig. 1). With their experiment Hanbury Brown and Twiss founded the discipline of quantum-statistical measurements, which has led to important developments in quantum optics (4). Correlation experiments along the lines of Hanbury Brown and Twiss are today also an important tool in particle physics (5).

In the Hanbury Brown and Twiss (HBT) experiment an average over many particles is measured. In general, the time-dependent intensity $I(t)$ of a stream of particles exhibits fluctuations $\Delta I$ around the average current $\langle I\rangle$ known as shot noise, which has recently attracted much attention in electrical transport of submicrometer-sized nanostructures $(6,7)$. Shot noise is due to the fluctuation $\Delta N$ of the number of particles $N$ detected during a certain time interval. For a classical stream obeying Poisson statistics $\left\langle(\Delta N)^{2}\right\rangle=\langle N\rangle$, whereas for a stream of bosons obeying Bose-Einstein statistics $\left\langle(\Delta N)^{2}\right\rangle$ is larger, that is, super-Poissonian. In contrast, for degenerate fermions the stream is noiseless, indicating the complete absence of fluctuations in the occupation of states according to the Pauli principle. Suppression of electrical-current noise of this origin has recently been found in electrical measurements on quantum-point 


\section{R E P O R T S}

contacts and nanowires $(8-11)$.

The HBT experiment measures, instead of the fluctuations in a single beam, the correlations between the transmitted and reflected partial beams originating from a beam splitter. At a single-particle level the outcome of such an experiment is obvious. If it is known that there is only one particle in the measuring apparatus, the particle is either detected in the transmitted or in the reflected channel. Hence, the signals from the two detectors are fully anticorrelated, independent of whether the particle is a boson or a fermion. The situation is, however, different if we consider the mean intensity fluctuations in a beam whose initial state is not prepared at a single-particle level. Now, a correlator measuring $\left\langle\Delta I_{\mathrm{t}} \Delta I_{\mathrm{r}}\right\rangle$, where $\Delta I_{\mathrm{t}, \mathrm{r}}$ are the intensities in the transmitted ( $\mathrm{t}$ ) and reflected (r) beams, is predicted to yield a positive signal in the case of Bose-Einstein statistics (the HBT result), no correlations in the classical case, and a negative sign (anticorrelation) in the case of Fermi-Dirac statistics. While negative correlations have been observed for photon fields with special statistics (12), an HBTtype intensity-correlation experiment has not yet been carried out for a nonclassical partially degenerate stream of fermions. Although such an experiment was proposed to measure fermionic correlations of electrons in a free electron beam (13), the experiment is difficult to perform because the expected correlations are very small due to the low electron density for which quantum statistics barely matters. Two successful realizations of the HBT experiment for a beam of fermions is also reported in this issue (14). Both are based on semiconductor devices in which a degenerate beam of electrons can conveniently be obtained. The HBT experiment and other more advanced multiterminal correlation experiments have been considered theoretically before $(6,15-18)$. In an elegant study, Liu et al. have explored quantum-statistical properties of a twobeam collision experiment using a semiconductor multiterminal nanostructure (9).

A convenient way to realize a well-defined electron beam with a tunable beam splitter has been discussed by Büttiker (6). It is based on the edge-channel picture for electron transport in a two-dimensional electron gas in the quantum Hall regime (19). A lithographically patterned metallic gate serves as a tunable beam splitter for the incoming electrons (Fig. 2). A voltage source $V$ connected to reservoir 1 injects the charge current $I$ into the Hall bar. In the quantum Hall regime the current is confined to one-dimensional edge channels (shown in Fig. 2 as lines along the edge of the sample with arrows indicating the direction of the current flow). The injected electrons travel along the upper edge-channel until reaching the split gate, where they are either transmitted with probability $t$ to leave the sample at contact 2 , or reflected leaving at contact 3 (contact 4 is not connected and is irrelevant for this type of experiment). In the case of zero magnetic field, where no edge channels are formed, it would be impossible to separate the incoming from the reflected beam. Provided $\mathrm{eV}$ $\gg k T$, the transmitted and reflected currents $I_{\mathrm{t}, \mathrm{r}}$ and their fluctuations $\Delta I_{\mathrm{t}, \mathrm{r}}$ are measured at contact 2 and 3 . The theory predicts for the spectral densities of the auto- and cross-correlations, that is, the current correlations normalized to unit frequency bandwidth (16)

$$
\left\langle\Delta I_{\alpha} \Delta I_{\beta}\right\rangle_{\mathrm{s}}= \pm 2 e|I| t(1-t)
$$

with $e$ the electron charge quantum and $\alpha, \beta$ either $t$ or $r$. The positive sign corresponds to $\alpha=\beta$ (autocorrelation, that is, conventional noise) and the negative one to $\alpha \neq \beta$ (crosscorrelation). Hence, the fluctuations of the reflected and transmitted beams are predicted to be anticorrelated with a magnitude that equals the autocorrelation. This is the largest possible correlation corresponding to fully anticorrelated partial beams, that is, $\Delta I_{\mathrm{t}}=$ $-\Delta I_{\mathrm{r}}$. From Eq. 1 it is clear that a channel with zero transmission $(t=0)$ or with full transmission $(t=1)$ is noiseless, whereas the noise is largest if $t=1 / 2$.

The experiments reported here are all conducted in magnetic field corresponding to filling factor $v=2$. Because the spin-splitting of Landau levels is not resolved in transport measurements, one (spin-degenerate) edge state is formed at $v=2$. The beam splitter is adjusted to transmit and reflect electrons with $50 \%$ probability $(t=0.5)$.

Figure 3 shows the cross-correlation (squares) of the fluctuations $\Delta I_{\mathrm{t}}$ and $\Delta I_{\mathrm{r}}$ versus bias current $I$ at $T=2.5 \mathrm{~K}$. For this

Fig. 1. The particles in a beam of bosons obeying Bose-Einstein statistics tend to cluster (bunching). Consequently, a positive correlation is observed between two partial beams generated by a beam splitter. In contrast, in a degenerate beam of fermions the particles expel each other (antibunching) because a fermionic state can only be occupied once. Consequently, the partial beams are expected to
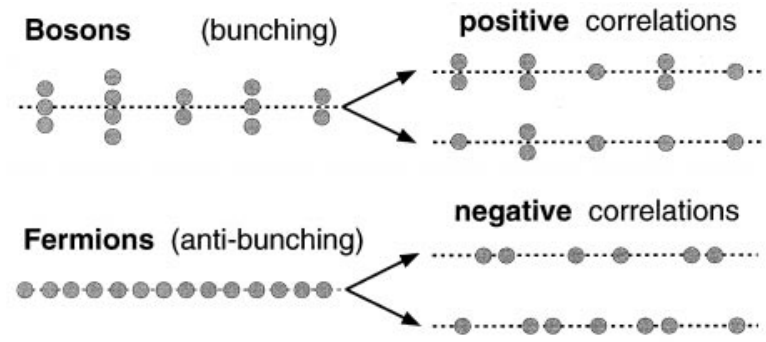
be fully anticorrelated.

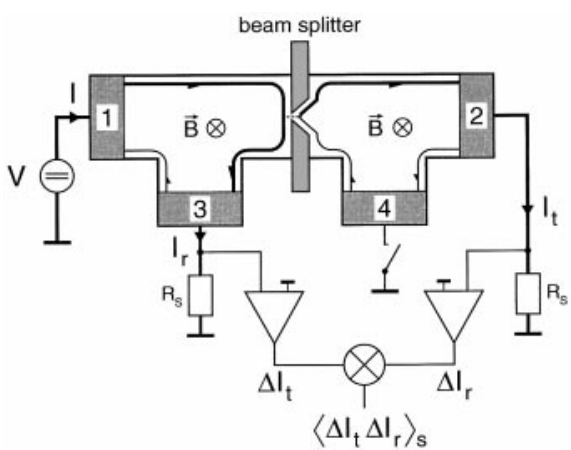

Fig. 2. Intensity correlation experiment for a degenerate beam of electrons realized in a semiconductor Hall bar connected to four electron reservoirs (dark shading). A metallic split gate (light shading) serves as a tunable beam splitter. The primary beam I originates from the electrons injected by the voltage source $V$ connected to reservoir 1 . These electrons move along the upper edge channel until reaching the gate, where they are either transmitted into contact 2 or reflected into 3 . The time-dependent transmitted and reflected currents $I_{\mathrm{t}, \mathrm{r}}$ are converted to voltage signals by two 1-kilohm series resistors $R_{\mathrm{s}}$ and then amplified. Finally, an electronic correlator determines the spectral correlations $\left\langle\Delta I_{t} \Delta I_{r}\right\rangle_{s}$ of the fluctuations $\Delta I_{t, r}$ at a central frequency in the range of $100 \mathrm{kHz}$ to $1 \mathrm{MHz}(11)$.

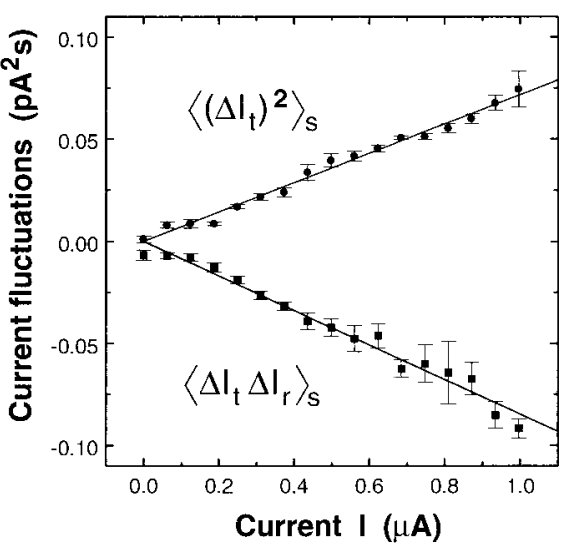

Fig. 3. Measured spectral densities of currentfluctuation correlations as a function of the current $I$ of the incident beam at temperature $T=2.5 \mathrm{~K}$ and with the beam splitter adjusted to $t=50 \%$ transmission. $\left\langle\left(\Delta / t_{t}\right)^{2}\right\rangle_{s}$ denotes the autocorrelation in the transmitted channel and $\left\langle\Delta l_{\mathrm{t}} \Delta I_{\mathrm{r}}\right\rangle_{\mathrm{s}}$ the cross-correlation between the transmitted and reflected channels. Currentindependent fluctuations, such as thermal noise and residual amplifier noise, have been subtracted. From the experiment we deduce for the absolute slope $0.23 \cdot 2 \mathrm{el}$ and $0.26 \cdot 2 \mathrm{el}$ for the autocorrelation and cross-correlation, respectively. This is in good agreement with the expected prefactor given by $t(1-t)=1 / 4$. 


\section{RE P O R T S}

experiment thermal noise can be neglected, because $e V \gg k T$. Accordingly, a nearly linear dependence with a negative slope is found, proving that the fluctuations are indeed anticorrelated. Moreover, the autocorrelation (circles) of the transmitted current $\left\langle\left(\Delta I_{\mathrm{t}}\right)^{2}\right\rangle_{\mathrm{s}}$ and the reflected current (not shown) have a positive slope, which in magnitude is equal to the slope of the measured cross-correlation, as predicted by Eq. 1. Thus, the experiment confirms that the partial beams are fully anticorrelated.

As emphasized above, a beam-splitter intensity correlation experiment always results in fully anticorrelated partial beams independent of the statistics, only if the experiment is prepared in such a way that there is no uncertainty in the occupation of the initial beam. The observed full anticorrelation can therefore be taken as a proof that the incident beam is occupied with unit probability and, hence, is noiseless by virtue of the Pauli principle. This also follows formally from $\left\langle(\Delta I)^{2}\right\rangle=\left\langle\left(\Delta I_{\mathrm{t}}+\Delta I_{\mathrm{r}}\right)^{2}\right\rangle=\left\langle\left(\Delta I_{\mathrm{t}}\right)^{2}\right\rangle+$ $\left\langle\left(\Delta I_{\mathrm{r}}\right)^{2}\right\rangle+2\left\langle\Delta I_{\mathrm{t}} \Delta I_{\mathrm{r}}\right\rangle$, which equals zero to within experimental accuracy.

Alternatively, the experiment demonstrates that a constant current emanates from the injector contact 1 held at constant electrochemical potential. This is a fundamental requirement of the Landauer-Büttiker theory of transport in mesoscopic systems (20). An applied voltage $V$ injects a constant current $e^{2} V / h$ into each accessible propagating onedimensional channel (here the edge states),

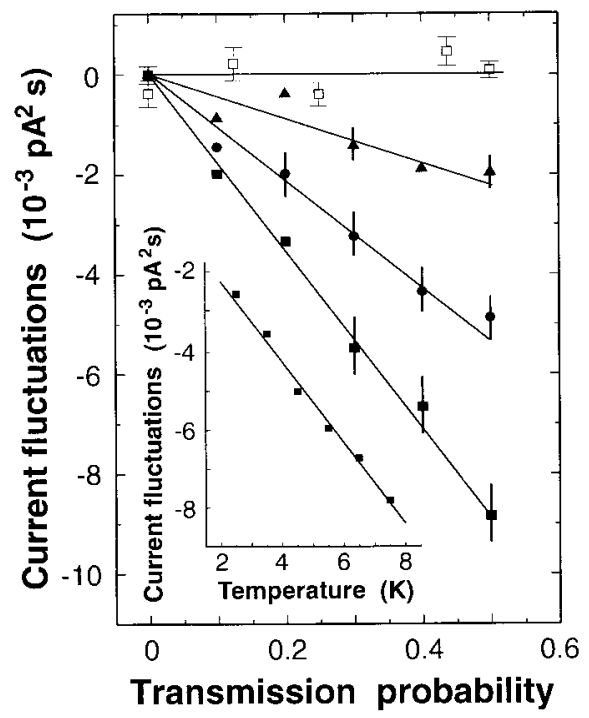

Fig. 4. Measured spectral densities of correlations between equilibrium fluctuations $(V=0)$ as a function of transmission probability $t$ for the device shown in Fig. 2 with contact 4 open (solid symbols) and contact 4 closed (open symbols). The temperatures are from top to bottom 7.5, 2.5, 5.0, and $7.5 \mathrm{~K}$. (Inset) The correlations as a function of temperature for $t=0.5$. The measured values have been corrected for the thermal noise originating from the series resistors $R_{\mathrm{s}}$. thereby converting the voltage source into a perfect current source. Our experiment may therefore be taken as a confirmation of the edge-state picture for the integer quantumHall regime (19).

Up to now the experiments were in the regime $\mathrm{eV} \gg k T$, where thermal fluctuations are negligible. Can thermal fluctuations at two different reservoirs be correlated as well? From (16) we expect for the equilibrium correlations between two different reservoirs $\alpha$ and $\beta$

$$
\left\langle\Delta I_{\alpha} \Delta I_{\beta}\right\rangle_{\mathrm{s}}=-2 k T G_{0}\left(t_{\alpha \beta}+t_{\beta \alpha}\right)
$$

where $G_{0}=2 e^{2} / h$ is the quantum conductance and $t_{\beta \alpha}\left(t_{\alpha \beta}\right)$ the (direct) transmission probability from contact $\alpha$ to $\beta$ ( $\beta$ to $\alpha$ ). This expression predicts that thermal fluctuations at different contacts are also anticorrelated, provided $t_{\beta \alpha}$ or $t_{\alpha \beta}$ are nonzero. For the equilibrium case, however, the negative sign is not specific to the statistics; rather, it is a consequence of particle conservation. In a multiterminal device different contacts may not be coupled with a finite direct transmission probability, leaving thermal noise of these contacts uncorrelated (16).

We first consider the device in Fig. 2 with contact 4 open. Although formally there is no direct transmission from contact 2 to 3 , the fluctuating current transmitted from contact 2 into contact 4 is reinjected unaltered again by this contact because contact 4 is open. For this reason, contact 4 can be disregarded and $t_{32}$ can be taken to be equal to $t$. In the opposite direction the transmission vanishes $\left(t_{23}=0\right)$, because fluctuations originating from contact 3 are terminated by contact 1 , which is connected to ground. We therefore expect

$$
\left\langle\Delta I_{\mathrm{t}} \Delta I_{\mathrm{r}}\right\rangle_{\mathrm{s}}=-2 k T G_{0} t
$$

The fluctuations are now proportional to the transmission probability $t$ and to the temperature $T$. The measured correlations are shown in Fig. 4 for three different temperatures (solid symbols). As predicted, negative correlations proportional to the transmission probability $t$ are observed. The inset shows the temperature dependence of the correlations at $t=0.5$. The measured magnitudes agree with theory within the measurement accuracy. In a control experiment contact 4 has been connected to ground. This cancels out any direct transmission from contact 2 to 3 and therefore destroys the correlation. The measurement in Fig. 4 (open squares) obtained for a relatively large temperature of $T=7.5 \mathrm{~K}$ confirms this expectation. Although there is an equilibrium fluctuating current at both contacts 2 and 3 , these currents fluctuate independently (16).

Correlations between current fluctuations are sensitive to the statistics of the charge degree of freedom. Having demonstrated the anticorrelation expected for electrons obeying Fermi-Dirac statistics, it would be interesting to extend this work to unconventional electron states. An example is the fractional Hall state, which is described by fractional statistics (21). It also appears possible to demonstrate bunching with electrons, because electron streams showing fluctuations exceeding the classical Poisson value have recently been observed in resonant tunneling devices and in superconducting weak links (22).

\section{References and Notes}

1. R. Hanbury Brown and R. Q. Twiss, Philos. Mag. Ser. 7 45, 663 (1954).

2. ㄴ. Nature 177, 27 (1956); ibid. 178, 1046 (1956); E. Purcell, ibid., p. 1449.

3. B. L. Morgan and L. Mandel, Phys. Rev. Lett. 16, 1012 (1966).

4. For a review, see R. Loudon, Rep. Prog. Phys. 43, 913 (1980).

5. For a review, see G. Baym, nucl-th/9804026.

6. M. Büttiker, Phys. Rev. Lett. 65, 2901 (1990)

7. For recent reviews, see M. J. M. de Jong and C. W. J. Beenakker, in Mesoscopic Electron Transport, L. P. Kouwenhoven, G. Schön, L. L. Sohn, Eds. (NATO ASI Series E, no. 345, Kluwer, Dordrecht, Netherlands, 1996), pp. 225-258; M. Reznikov et al., Superlattices Microstruct. 23, 901 (1998).

8. M. Reznikov, M. Heiblum, H. Shtrikman, D. Mahalu, Phys. Rev. Lett. 75, 3340 (1995); A. Kumar, L. Saminadayar, D. C. Glattli, ibid. 76, 2778 (1996).

9. R. C. Liu, B. Odom, Y. Yamamoto, S. Tarucha, Nature 391, 263 (1998); see also R. Landauer, ibid. 392, 658 (1998); M. Büttiker, Phys. World (March 1998), p. 30.

10. A. Steinbach, J. M. Martinis, M. H. Devoret, Phys. Rev. Lett. 76, 3806 (1996); R. J. Schoelkopf, P. J. Burke, A. A. Kozhevnikov, D. E. Prober, ibid. 78, 3370 (1997).

11. M. Henny, S. Oberholzer, C. Strunk, C. Schönenberger, Phys. Rev. B. 59, 2871 (1999).

12. H. J. Kimble, M. Dagenais, L. Mandel, Phys. Rev. Lett. 39, 691 (1977); P. Grangier, G. Roger, A. Aspect, Europhys. Lett. 1, 173 (1986); F. Diedrich and H. Walther, Phys. Rev. Lett. 58, 203 (1987).

13. M. P. Silverman, Phys. Lett. A 120, 442 (1987); T. Kodama et al., Phys. Rev. A 57, 2781 (1998).

14. Oliver el al. have realized a similar HBT experiment independently [W. D. Oliver, J. Kim, R. C. Liu, Y. Yamamoto, Science 284, 299 (1999)].

15. Th. Martin and R. Landauer, Phys. Rev. B 45, 1742 (1992).

16. M. Büttiker, ibid. 46, 12485 (1992).

17. R. C. Liu and Y. Yamamoto, ibid. 49, 10520 (1994).

18. Ya. M. Blanter and M. Büttiker, ibid. 56, 2127 (1997); E. V. Sukhorukov and D. Loss, Phys. Rev. Lett. 80, 4959 (1998); T. Gramespacher and M. Büttiker, ibid. 81, 2763 (1998).

19. For a review, see M. Büttiker, in Semiconductors and Semimetals: Nanostructured Systems, M. Reed, Ed. (Academic Press, New York 1992), vol. 35, pp. 191-277.

20. R. Landauer, IBM J. Res. Dev. 32, 306 (1988); M. Büttiker, ibid., p. 317; R. Landauer, Phys. Scr. T42, 110 (1992); Y. Imry, in Directions in Condensed Matter Physics, G. Grinstein and G. Mazenko, Eds. (World Scientific, Singapore 1986), pp. 101-163; C. W. J. Beenakker and H. van Houten, in Solid State Physics, H. Ehrenreich and D. Turnbull, Eds. (Academic Press, New York, 1991), pp. 1-228.

21. L. Saminadayar, D. C. Glattli, Y. Jin, B. Etienne, Phys. Rev. Lett. 79, 2526 (1997); R. de-Picciotto et al., Nature 389, 162 (1997).

22. G. Innaccone, G. Lombardi, M. Macucci, B. Pellegrini, Phys. Rev. Lett. 80, 1054 (1998); P. Dieleman et al., ibid. 79, 3486 (1997); T. Hoss et al., cond-mat/ 9901129.

23. We thank M. Büttiker, D. C. Glattli, and T. Gramespacher for useful comments. This work is supported by the Swiss National Science Foundation.

30 November 1998; accepted 1 March 1999 